\title{
Surface Plasmon - Guided Mode strong coupling
}

\author{
Aurore Castanié*, Didier Felbacq and Brahim Guizal
}

\author{
University of Montpellier 2 \\ Laboratory Charles Coulomb UMR CNRS-UM2 5221 \\ *E-mail: aurore.castanieduniv-montp2.fr
}

\begin{abstract}
It is shown that it is possible to realize strong coupling between a surface plasmon and a guided mode in a layered structure. The dispersion relation of such a structure is obtained through the S-matrix algorithm combined with the Cauchy integral technique that allows for rigorous computations of complex poles. The strong coupling is demonstrated by the presence of an anticrossing in the dispersion diagram and simultaneously by the presence of a crossing in the loss diagram. The temporal characteristics of the different modes and the decay of the losses in the propagation of the hybridized surface plasmons are studied.
\end{abstract}

\section{Introduction}

A surface plasmon (SP) is a transverse magnetic (TM)polarized electromagnetic wave coupled to the collective longitudinal oscillations of the free electron density existing near the surface of metal [1]. The requirement for the existence of a SP is that the metal in contact with the dielectric must have a relative permittivity whose real part is negative at the frequency of light which will be used to excite the SP.

Besides, emission through SPs is very efficient deexcitation mechanism for emitting materials close to a metallic surface. Indeed, the modification of the spontaneous emission of molecules situated near a metal surface with regard to their emission in the space was experimentally demonstrated by K. H. Drexhage and al. [2]. Furthermore, in 1999, P. T. Worthing and al. [3] etablished that the mode of deexcitation privileged for ions $\mathrm{Eu}^{3+}$ close to a metallic surface are the SPs modes. These results were obtained in a weak coupling regime. The dynamics of the excitement is then irreversible and the probability of the presence of the transmitter in the incited state decreases exponentially. SPs were extensively studied in this regime.

When the interaction between an electronic level and an electromagnetic mode increases, it can enter in a regime of strong coupling. This regime was studied in an extensive way in optical microcavities since the works of C. Weisbush and al. [4]. More recently, it was demonstrated between SPs and excitons in organic semiconductors [5, 6, 7]. It is a phenomenon that is interesting both from the point of view of fundamental quantum physics as well as from the point of view of technology.

In the present work, we investigate the strong coupling between a SP and a guided mode in a multilayered planar

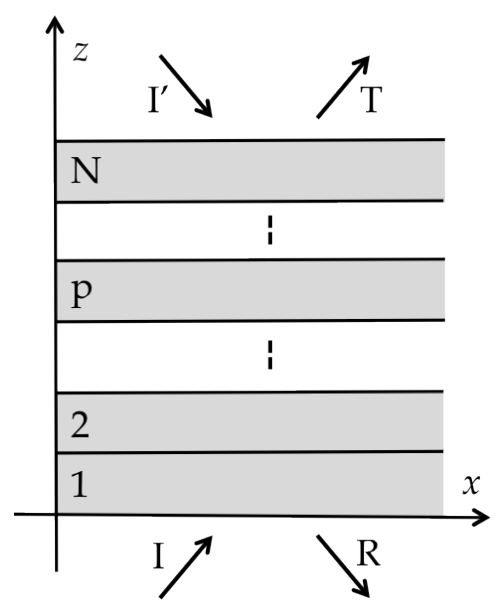

Figure 1: General scheem of a multilayered structure.

structure. First of all, the determination of the poles [8] of the S-matrix determinant allows to draw the complex dispersion relation of the structure. Moreover, we used a finite difference time domain (FDTD) algorithm for frequency dependant media which reveals temporal characteristics of the different modes [9].

\section{Theory}

It is well known that SPs only exist under TM-polarization. We consider a multilayered structure with $N$ films with dielectric permittivities $\varepsilon_{p}$. In this case, the transverse magnetic field can be reprensented by :

$$
\left\{\begin{array}{l}
u_{0}(x, z)=I e^{i\left(\alpha x+\beta_{0} y\right)}+R e^{i\left(\alpha x-\beta_{0} y\right)} \\
u_{p}(x, z)=a_{p} e^{i\left(\alpha x+\beta_{p} y\right)}+b_{p} e^{i\left(\alpha x-\beta_{p} y\right)} \\
u_{N}(x, z)=T e^{i\left(\alpha x+\beta_{N} y\right)}+I^{\prime} e^{i\left(\alpha x-\beta_{N} y\right)}
\end{array}\right.
$$

For each medium, $\alpha^{2}+\beta_{p}^{2}=k_{0}^{2} \varepsilon_{p} \mu_{0}$. The constants $I$, $I^{\prime}, R, T, a_{p}$ and $b_{p}$ are the amplitudes of the different waves (figure 1). The boundary conditions lead to the system of equations we have to solve with the formalism of the Smatrix.

\subsection{The S-matrix formalism}

The S-matrix formalism allows to find the coefficients of reflexion and transmission $R$ and $T$ but also all the modes 
of the structure. Indeed, it relates the outgoing amplitudes, $R$ and $T$, to the incoming ones, $I$ and $I^{\prime}$ :

$$
\left(\begin{array}{c}
R \\
T
\end{array}\right)=S(\omega, \alpha)\left(\begin{array}{c}
I \\
I^{\prime}
\end{array}\right)
$$

The modes of the structure are then defined by the existence of outgoing waves in the absence of excitement. With $O U T=(R, T)$, this condition is translated by $S^{-1} O U T=0$. Thus, to find the modes of the structure, for each frequency $\omega$, we look for the propagation constant $\alpha$ which correspond to the cancellation of the determinant of the matrix $S^{-1}$ or in an equivalent way to the maximization of the determinant of the S-matrix. In other words, we have to find the poles of $\operatorname{det} S(\omega, \alpha)$. For each $\omega$, we look for the corresponding poles $\alpha_{0}$ in the complex plane by using Cauchy integrals :

$$
\alpha_{0}=\int_{\gamma} \alpha S(\omega, \alpha) d \alpha / \int_{\gamma} S(\omega, \alpha) d \alpha
$$

Where $\gamma$ is a closed loop of the complex plane containing the seeked poles. This method enables to obtain the dispersion and loss diagrams corresponding to the studied structure.

\subsection{Finite Difference Time Domain method}

A model based on the two-dimensional FDTD method with frequency dependent media is used [10]. We consider an isotropic and non-magnetic multilayered structure (fig. 2) with dielectric functions $\varepsilon_{1}, \varepsilon_{2}, \varepsilon_{g}$ and $\varepsilon_{m}(\omega)=\varepsilon_{\infty}-$ $\omega_{p} /(\omega(\omega+i \gamma))$; where $\omega_{p}$ is the plasma frequency and $\gamma$ the damping frequency [11]. With $\varepsilon_{0}$ and $\mu_{0}$ egal to 1 , we have the constitutive relations $\mathbf{B}=\mathbf{H}$ and $\mathbf{D}=\varepsilon_{m}(\omega) \mathbf{E}$. Therefore, the relationship between $\mathbf{D}$ and $\mathbf{E}$ becomes a convolution in the FDTD algorithm. With the $\mathrm{Z}$ transform, it's possible to replace discrete convolution with multiplications [12], so that the constitutive relation for $\mathbf{D}$ and $\mathbf{E}$ becomes :

$$
D(z)=\varepsilon_{m}(z) E(z) \Delta t,
$$

where $\Delta t$ is the time step used in FDTD and $\varepsilon_{m}(z)$ corresponds to $\varepsilon_{m}(\omega)$ in $z$-space :

$$
\varepsilon_{m}(z)=\frac{\varepsilon_{\infty}}{\Delta t}+\frac{\omega_{p}^{2}}{\gamma}\left(\frac{1}{1-z^{-1}}-\frac{1}{1-z^{-1} e^{-\gamma \Delta t}}\right) .
$$

\section{Complex dispersion relation}

We consider the structure in figure 2 which is a stratified medium containing a dielectric waveguide near a metallic film, the whole being embedded between two dielectric hosts and separated by a dielectric layer of thickness $e$. This structure supports three different modes : a guided mode (GM) which lives in the medium with $\varepsilon_{g}$ and two SPs over the interfaces of the metal. For this simulations, the permittivity of the metal is taken from the experimental results of E. Palik [13]. All the permittivities values was chosen to

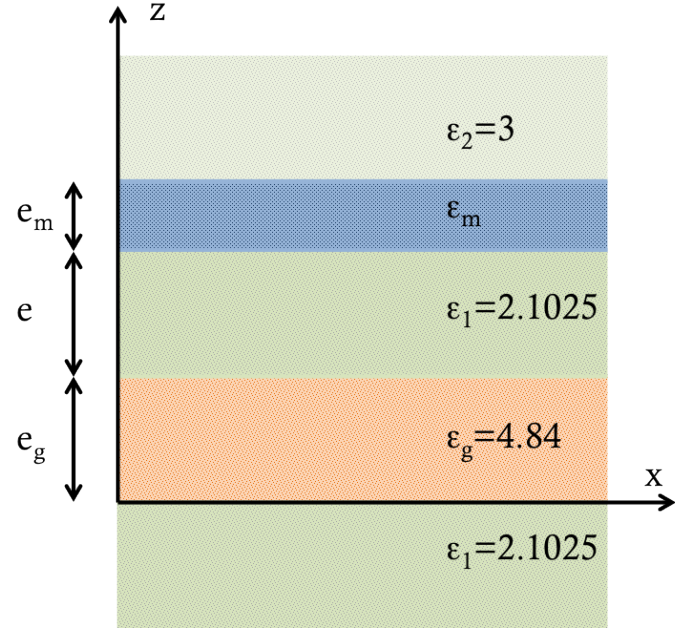

Figure 2: Multilayered structure with a dielectric waveguide $\left(\varepsilon_{g}\right)$ with $e_{g}=130 \mathrm{~nm}$ and a metallic film $\left(\varepsilon_{m}\right)$ with $e_{m}=45 \mathrm{~nm}$ inserted between two dielectrics hosts ( $\varepsilon_{1}$ and $\varepsilon_{2}$ ) and separated by a dielectric layer. The thickness $e$ is the parameter which is going to change in the simulations.

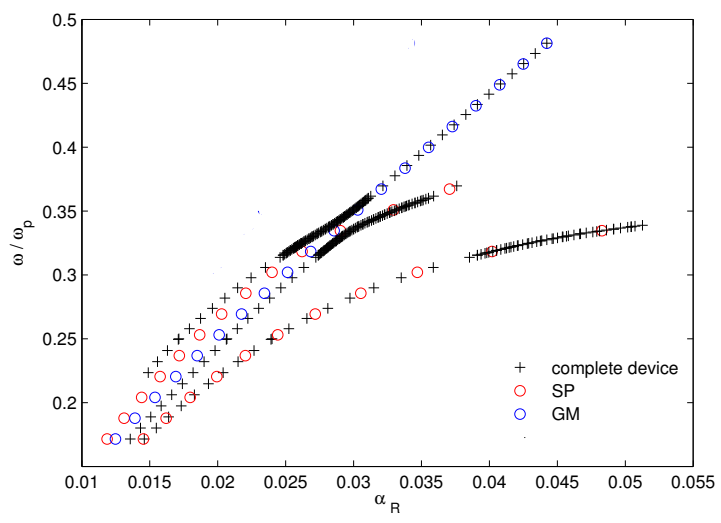

Figure 3: Dispersion relation for a thickness $e=98 \mathrm{~nm}$ $:$ the normalized frequency (to the plasma frequency $\omega_{p}$ ) in function of the real part of the propagation constant $\alpha$. Taken separately, the modes have the dispersion relations in blue and red.

allow the manufacturing of this structure to test experimentally our results [14].

To reveal the interactions between these modes, we draw the dispersion relations obtained for this structure with the research of the poles of the determinant of the S-matrix. The results are summarized is the figures 3 and 4 .

In figure 3, we note the presence of an anticrossing of the dispersion curves (black points) when for the same values of the frequency and the propagation constant, we have a crossing in the uncoupled case (blue and red points). This anticrossing arround $\alpha_{R}=0.29$ is the result of hybridization of the guided mode and the SP which lives below the metallic layer, closer to the waveguide. This is the first proof of the strong coupling between these modes. 


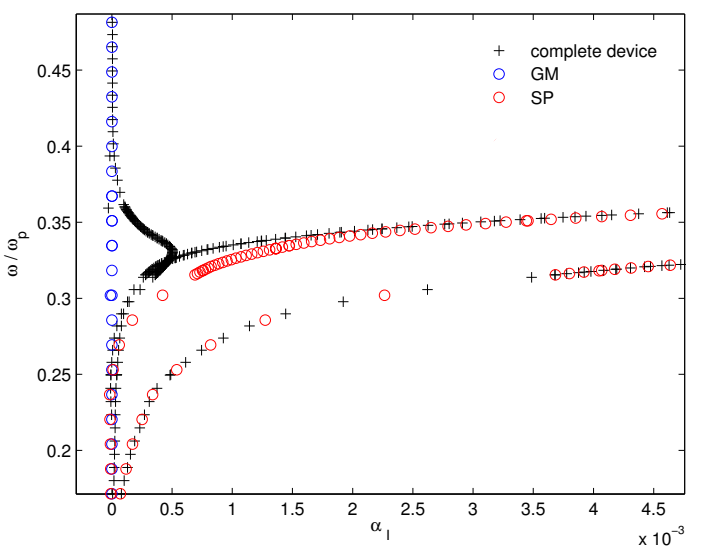

Figure 4: Loss diagram for a thickness $e=98 \mathrm{~nm}$ : the normalized frequency (to the plasma frequency $\omega_{p}$ ) in function of the imaginary part of $\alpha$. Separately, the absorption of each mode is in blue and red.

Another way we can illustrate this strong coupling is to consider the curves of the loss diagram in the figure 4 . Once again, the blue and ref points are relative to the uncoupled case. The loss diagram reveal the decay of the losses for the SP below the metallic layer. Indeed, whereas the losses for the guided mode increase, those for the SP decrease until obtaining the crossing of the curves : there is an exchange of energy between the guided mode and the plasmon.

Then, it's possible to look at the spatial structure of the hybrid mode. When we excite the structure at the wavelength $\lambda=409 \mathrm{~nm}$ which corresponds to the anticrossing of the curves, the mode oscillates between the waveguide and the lower interface of the metallic layer. These oscillations are spatial Rabi oscillations with a period bigger than the micrometer.

\section{Temporal oscillations}

The FDTD allows to study evolution of the structure energy according to time by exciting the structure with a sinusoidal beam. Figure 5 shows oscillations of the field intensity between the center of the waveguide (in black) and the metal-dielectric interface (in red). For this simulations, the permittivity of the metal is taken from equation (5). After the transient regime, the permanent regime takes place at roughly $t=9$ fs.

In this case, these oscillations can called temporal Rabi oscillations. To obtain a realistic result, we can add a gaussian envelope at the beam. The temporal oscillations decay in time but the life time of the SP is higher than the uncoupled case. The detailed characterization of these oscillations is the next step of our work.

Spatial and temporal oscillations between these modes illustrate the transfert of MG energy towards the SP mode and are associated with the decay of the losses for this last mode.

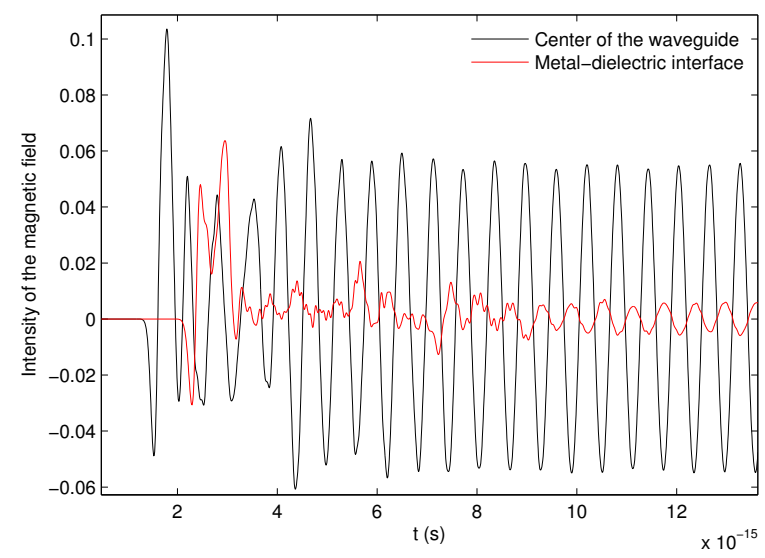

Figure 5: Temporal oscillations between the MG (in black) and the SP under the metal (in red) with a sinusoidal incident beam.

\section{Conclusion}

The existence of a strong coupling regime between SP and a guided mode in a multilayered structure was demonstrated. The numerical tools used allow to obtain a rigorous computation of the dispersion relation and loss diagram. The FDTD algorithm reveals temporal oscillations between these modes.

This study is a first step towards the realization of a transfert of energy between these modes like in Spaser $[15,16]$.

\section{Acknowledgement}

The financial support of the Agence Nationale de la Recherche under the project OPTRANS is gratefully acknowledged.

\section{References}

[1] H. Raether, Surface plasmons on smooth and rough surfaces and on gratings, Springer Verlag, Berlin 1988.

[2] K. H. Drexhage, Interaction of light with monomolecular dye layers, Progress in Optics, 12, E. Wolf ed., North-Holland publishing, 1974.

[3] P. T. Worthing, R. M. Amos, W. L. Barnes, Modification of the spontaneous emission rate of $\mathrm{Eu}^{3+}$ ions embedded within a dielectric layer above a silver mirror, Phys. Rev. A, 59, 865, 1999.

[4] C. Weisbuch, M. Nishioka, A. Ishikawa, Y. Arakawa, Observation of the coupled exciton-photon mode splitting in a semiconductor quantum microcavity, Phys. Rev. Lett., 69 (23), 3314, 1992. 
[5] J. Bellessa, C. Symonds, K. Vynck, A. Lemaitre, A. Brioude, L. Beaur, J. C. Plenet, P. Viste, D. Felbacq, E. Cambril, P. Valvin, Giant Rabi splitting between localized mixed plasmon-exciton states in a two-dimensional array of nanosize metallic disks in an organic semiconductor, Phys. Rev. B., 80, 033303, 2009.

[6] J. Bellessa, C. Bonnand, J. C. Plenet, Strong coupling between surface plasmons and excitons in an organic semiconductor, Phys. Rev. Lett., 93, 036404, 2004.

[7] R. L. Chern, D. Felbacq, Artificial magnetism and anticrossing interaction in photonic crystals and splitring structures, Phys. Rev. B, 79, 075118, 2009.

[8] D. Felbacq, Poles and zeros of the scattering matrix associated with defect modes, J. Phys. A : Math. Gen., 33, 7137, 2000.

[9] A. Taflove, S. C. Hagness, Computational electrodynamics : The finite difference time domain method, Artech House, 2nd ed., 2000.

[10] A. Mohammadi, M. Agio, Dispersive contour-path finite-difference time-domain algorithm for modelling surface plasmon polaritons at flat interfaces, Optics Express, 14 (23), 11330, 2006.

[11] M. Born, E. Worlf, Principles of Optics, Cambridge U. Press, 7th ed., 1999.

[12] D. M. Sullivan, Electromagnetic simulation using the FDTD method, IEEE Press, 2000.

[13] E. Palik, Handbook of optical constants of solids Academic Press, Inc., New York, 1985.

[14] J. Bellessa, S. Rabaste, J. C. Plenet, J. Dumas, J. Mugnier, O. Marty, $\mathrm{Eu}^{3+}$-doped microcavities fabricated by sol-gel process, Inc., New York, 1985.

[15] D. J. Bergman, M. I. Stockman, Surface plasmon amplification by stimulated emission of radiation: Quantum generation of coherent surface plasmons in nanosystems, Phys. Rev. Lett., 90, 027402, 2003.

[16] M. A. Noginov, G. Zhu, A. M. Belgrave, R. Bakker, V. M. Shalaev, E. E. Narimanov, S. Stout, E. Herz, T. Suteewong, U. Wiesner, Demonstration of a spaserbased nanolaser, Nature Letters, 460, 1110, 2009. 